\title{
Eigenvalue Analysis and Longtime Stability of Resonant Structures for the Meshless Radial Point Interpolation Method in Time Domain
}

\author{
Thomas Kaufmann, Student Member, IEEE, Christian Engström, \\ Christophe Fumeaux, Senior Member, IEEE, and Rüdiger Vahldieck, Fellow, IEEE
}

\begin{abstract}
A meshless collocation method based on radial basis function (RBF) interpolation is presented for the numerical solution of Maxwell's equations. RBFs have attractive properties such as theoretical exponential convergence for increasingly dense node distributions. Although the primary interest resides in the time domain, an eigenvalue solver is used in this paper to investigate convergence properties of the RBF interpolation method. The eigenvalue distribution is calculated and its implications for longtime stability in time-domain simulations are established. It is found that eigenvalues with small, but nonzero, real parts are related to the instabilities observed in time-domain simulations after a large number of time steps. Investigations show that by using global basis functions, this problem can be avoided. More generally, the connection between the high matrix condition number, accuracy, and the magnitude of nonzero real parts is established.
\end{abstract}

Index Terms-Eigenfunctions and eigenvalues, finite-difference methods, meshless methods, resonance, time-domain modeling.

\section{INTRODUCTION}

$\mathbf{I}$ $\mathrm{N}$ computational sciences, meshless methods recently gained attention as a versatile technique for solving numerical problems [1], [2]. For complex geometries in 3-D models, mesh generation is a computationally involved problem, and often requires additional manual labor to develop meshes producing good results. In contrast, meshless methods are based on an arbitrarily distributed set of node locations. This is an advantage since no explicit connectivity between these nodes is required, in contrast to the information required to store volumes, surfaces, and nodes in a conformal hexahedral or tetrahedral mesh-based method. The flexibility in the node distribution allows for conformal and multiscale modeling. Additionally adaptive refinement can be performed with a significantly smaller computational effort since nodes can be added, removed, or displaced with an overhead much smaller than conventional remeshing [3].

Manuscript received February 15, 2010; revised July 05, 2010; accepted July 20, 2010. Date of publication October 07, 2010; date of current version December 10, 2010. This paper is an expanded paper from the Asia-Pacific Microwave Conference, Singapore, December 7-10, 2009.

T. Kaufmann, C. Engström, and R. Vahldieck are with the Laboratory for Electromagnetic Fields and Microwave Electronics (IFH), ETH Zürich, 8092 Zürich, Switzerland (e-mail: thomas.kaufmann@ifh.ee.ethz.ch).

C. Fumeaux is with the School of Electrical and Electronic Engineering, The University of Adelaide, Adelaide SA 5005, Australia.

Color versions of one or more of the figures in this paper are available online at http://ieeexplore.ieee.org.

Digital Object Identifier 10.1109/TMTT.2010.2081250
Numerous approaches exist in the literature to solve the spatial interpolation problem, e.g., the smooth particle hydrodynamics (SPH) method [4], least squares based methods [5], or interpolation based on radial basis functions (RBFs) such as the radial point interpolation method (RPIM) [6], [7]. All these approaches have in common that a node-based interpolation scheme describes the connectivity between the nodes of the computational domain.

The method presented in this paper is a domain discretization collocation method with interpolations based on scalar RBFs. Most theoretical studies use global basis functions, which relate each node with all the others in the computational domain [8]. In [9], local basis functions have been introduced, which reduce the computational effort significantly, though at the cost of reduced accuracy and threat to numerical stability. The extent of the basis function is controlled by a shape parameter. It has been shown in [10] that the interpolation accuracy can be improved by using increasingly flat basis functions. This theoretically leads to exponential convergence by adapting the shape of the basis functions (c-refinement) only, at no additional computational cost for a fixed node distribution. There is, however, a practical limitation to this convergence, reached at the point where the problem becomes ill conditioned and the scheme breaks down. On the other side, increased spatial discretization ( $h$-refinement) is also expected to lead to a solution converging at a much higher rate than the conventional $h$ finite-element method (FEM) or finite difference time domain (FDTD). Combining both $c$ - and $h$-refinement allows for an adaptation scheme similar to $h p$-FEM [11], where a combination of mesh adaptation and local adaptation of the polynomial degree is used to increase accuracy. Here, we can combine the addition and movement of nodes with an optimization of the shape parameters ( $h c$-adaptivity). For scalar problems, a detailed study has been presented in [12] where an estimate for the spatial convergence rate for the maximum field error was given as $O\left(\beta^{n_{\text {dof }}^{1 / 2}}\right)$ with $0 \leq \beta \leq 1$ and $n_{\text {dof }}$ the number of degrees of freedom. This theoretical convergence rate is compared to that of FDTD and $h$-FEM in Fig. 1.

The research focus of the authors is on a time-domain implementation of RPIM applied to the solution of Maxwell's equations with emphasis on transient nonlinear phenomena in plasmonic and photonic nanostructures. In computational timedomain electromagnetics, recently demonstrated meshless approaches include different variants, such as SPH for electromagnetics based on SPH [13], [14] and the radial-point interpolation 


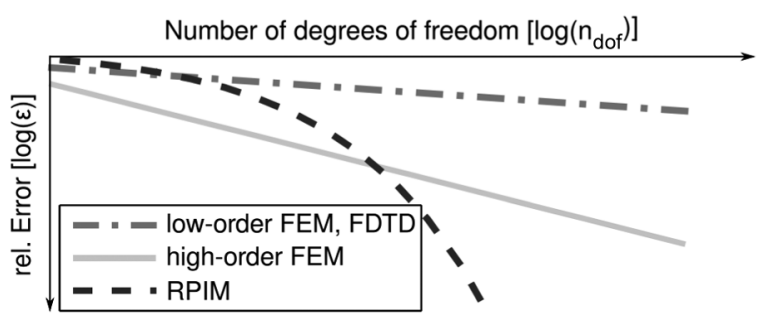

Fig. 1. Exemplified illustration of the highest theoretical convergence rates of relative errors when increasing the fineness of the spatial discretization. Shown are low-order methods like FEM or FDTD, high-order $h$-FEM, and RPIM using global RBFs.

time-domain (RPITD) method in [15]. An alternating-direction implicit implementation for RPITD has been presented in [16] and a 3-D implementation has been introduced in [17]. The occurrence of longtime instabilities for the method and the influence of parameter selection has been reported previously in [18]. However, recently the authors of [19] derived a stability criterion for $1-\mathrm{D}$ cases.

In [20], an eigenvalue solver for resonant structures has been introduced, based on Maxwell's equations using both electric and magnetic fields and their first-order spatial derivatives (firstorder form). The focus of this paper was on the convergence of the eigenvalues using local base functions. This paper extends the findings by further investigating the accuracy of the eigenmodes for numerous sets of parameters. A comparison between local and global basis functions is given and the possibility of spatial $(h)$ and parameter $(c)$ convergence is demonstrated. A detailed look at the eigenvalue distribution reveals the connection between longtime stability of the method and parameter selection. This paper focuses on a 2-D implementation, which is suitable for the general investigation of these effects. An extension in the future to three dimensions is a natural next step.

\section{RADIAL POINT INTERPOLATION}

RPIM uses an interpolation method based on RBFs. RBF interpolations have become the subject of considerable research efforts over recent years due to their excellent interpolation property for scattered data. In the following, both global and local basis functions are described and compared. Global basis functions take the whole domain into account. This leads to full matrices. In principle, the computational effort can be reduced by the use of compactly supported functions such as Wendland's functions [21]. A further decrease of computational cost is achieved in a localized implementation with local RBFs where only field values in the vicinity of each node inside a local support domain are considered, leading to an efficient algorithm using sparse matrices.

\section{A. Global Basis Functions}

A field component $u(\mathbf{x})$ at position $\mathbf{x}$ in a domain containing $N$ nodes is interpolated as

$\langle u(\mathbf{x})\rangle=\sum_{n=1}^{N} a_{n} r_{n}(\mathbf{x})+\sum_{m=1}^{M} b_{m} p_{m}(\mathbf{x})=\mathbf{r}(\mathbf{x})^{T} \mathbf{a}+\mathbf{p}^{T}(\mathbf{x}) \mathbf{b}$

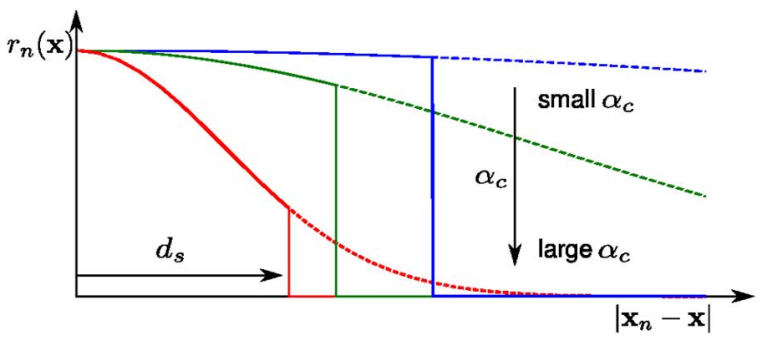

Fig. 2. Influence of the shape parameter $\alpha_{c}$ and support domain size $d_{s}$ on Gaussian RBFs. The solid lines shows a truncated Gaussian RBF defined locally in a support domain with radius $d_{s}$. The dashed lines show the untruncated Gaussian RBF on a global support domain.

where the RBFs

$$
r_{n}(\mathbf{x})=\exp \left(-\alpha_{c} \frac{\left|\mathbf{x}_{n}-\mathbf{x}\right|^{2}}{d_{c}^{2}}\right)
$$

are of Gaussian type with shape parameter $\alpha_{c}$ (Fig. 2). The normalization factor $d_{c}$ denotes the average distance to the next node inside the computational domain.

The interpolation by RBFs can be expanded to provide accurate approximation of polynomial functions (monomial reproduction) using monomial basis functions $p_{m}(\mathbf{x})$ in (1). Generally, those additional monomials are of low order

$$
\begin{aligned}
\text { zero order: } & \mathbf{p}^{T}=[], \quad M=0 \\
\text { first order: } & \mathbf{p}^{T}=[1, x, y], \quad M=3 \\
\text { second order: } & \mathbf{p}^{T}=\left[1, x, y, x^{2}, x y, y^{2}\right], \quad M=6
\end{aligned}
$$

with $M$ denoting the number of terms in the function $\mathbf{p}^{T}(\mathbf{x})$.

The interpolation parameters $\mathbf{a}$ and $\mathbf{b}$ are calculated in a preprocessing step as follows. A system is set up to interpolate the field values in the nodes $\mathbf{U}_{s}=\left(u_{1}, u_{2}, \ldots, u_{N}\right)^{T}$. This system can be written in the form

$$
\left(\begin{array}{c}
\mathbf{U}_{s} \\
\mathbf{0}
\end{array}\right)=\left(\begin{array}{cc}
\mathbf{R}_{0} & \mathbf{P}_{m} \\
\mathbf{P}_{m}^{T} & \mathbf{0}
\end{array}\right)\left(\begin{array}{l}
\mathbf{a} \\
\mathbf{b}
\end{array}\right)=\mathbf{G}\left(\begin{array}{l}
\mathbf{a} \\
\mathbf{b}
\end{array}\right)
$$

which includes the constraint condition $\mathbf{P}_{m}^{T} \mathbf{a}=\mathbf{0}$. This condition leads to a square invertible matrix. Thus, the interpolation parameters can be calculated as

$$
\left(\begin{array}{l}
\mathbf{a} \\
\mathbf{b}
\end{array}\right)=\mathbf{G}^{-1}\left(\begin{array}{c}
\mathbf{U}_{s} \\
\mathbf{0}
\end{array}\right)
$$

and the shape function $\Psi(\mathbf{x})$ is subsequently obtained from

$$
\begin{aligned}
\langle u(\mathbf{x})\rangle & =\left[\mathbf{r}^{T}(\mathbf{x}), \mathbf{p}^{T}(\mathbf{x})\right]\left(\begin{array}{l}
\mathbf{a} \\
\mathbf{b}
\end{array}\right) \\
& =\left[\mathbf{r}^{T}(\mathbf{x}), \mathbf{p}^{T}(\mathbf{x})\right] \mathbf{G}^{-1}\left(\begin{array}{c}
\mathbf{U}_{s} \\
\mathbf{0}
\end{array}\right) \\
& =\Psi(\mathbf{x}) \mathbf{U}_{s} .
\end{aligned}
$$

Note that $\Psi(\mathbf{x})$ fulfills the delta property, i.e., it guarantees an exact fit on the nodes. Similarly, the approximation of the spatial derivations along $\kappa=x, y$ can be expressed as

$$
\left\langle\partial_{\kappa} u(\mathbf{x})\right\rangle=\left[\partial_{\kappa} \mathbf{r}^{T}(\mathbf{x}), \partial_{\kappa} \mathbf{p}^{T}(\mathbf{x})\right] \mathbf{G}^{-1}\left(\begin{array}{c}
\mathbf{U}_{s} \\
\mathbf{0}
\end{array}\right)=\partial_{\kappa} \Psi(\mathbf{x}) \mathbf{U}_{s} .
$$




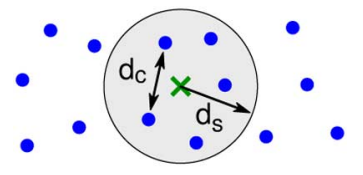

Fig. 3. Local support domain with radius $d_{s}$. Average node distance $d_{c}$ is used for normalization of RBFs.

The interpolation of scattered data using RBFs has only recently been investigated intensively, and the theoretical mathematical foundation is not completed yet. The question of whether the problem (4) is solvable and always yields unique solutions has only been partially answered [7] since there exist cases using local basis functions and monomial basis functions of order $M>0$ where the matrix is singular even though using positive definite RBFs. Nevertheless, it has been reported that for practical applications and using Gaussian basis functions, the system is solvable [21], [22].

The shape parameter $\alpha_{c}$ in (2) influences both the condition number of the matrix $\mathbf{G}$ and the interpolation accuracy. As mentioned previously, it has been shown that low values for $\alpha_{c}$, i.e., flat basis functions, give better interpolation accuracy. Considering the form of the matrix $\mathbf{G}$ in (4), it is understandable that the condition number of $\mathbf{G}$ becomes higher when the basis functions become flatter, as all elements in $\mathbf{R}_{0}$ become close to unity. At one point, even double precision arithmetic is bound to suffer from round-off errors that results in a significant degradation of the interpolation accuracy. In [23] and [24], this behavior has been described and several preconditioning techniques have been proposed. A study on the influence of the shape parameter $\left(\alpha_{c}\right)$ and the order of the monomial basis $(M)$ on the accuracy and eigenvalue distribution will be shown in the numerical experiments. Moreover, an algorithm to automatically select an optimized shape parameter is applied.

\section{B. From Global to Local Basis Functions}

The step from global to local basis functions is thoroughly described in [7], and therefore, only briefly summarized here. Instead of calculating the global basis functions for all nodes, only points within a support domain $\left|\mathbf{x}_{n}-\mathbf{x}\right|<d_{s}$ are considered to define local RBFs, as shown in Fig. 3 . The average node distance $d_{c}$ in 2-D can be approximated with knowledge of the number of neighbor nodes $\left(n_{A_{s}}\right)$ in the support domain as

$$
d_{c}=\frac{\sqrt{A_{s}}}{\sqrt{n_{A_{s}}}-1} .
$$

The term $A_{s}=\pi d_{s}^{2}$ is the physical area of the support domain with radius $d_{s}$. In the local case, this support domain size is an additional interpolation parameter, which has to be set as a tradeoff between efficiency and accuracy. The size of the support domain determines the number of nodes $\left(n_{A_{s}}\right)$ influencing a given node. Instead of computing one large global system, a large number of small local systems are then solved. As a result, the memory usage and calculation time can be heavily reduced

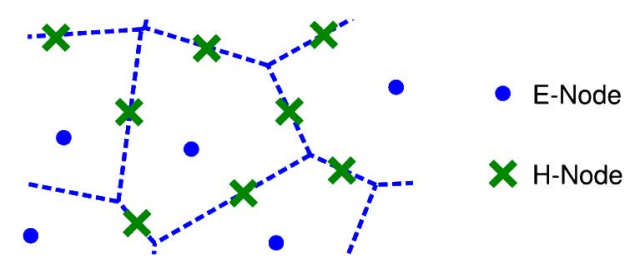

Fig. 4. Generation of dual $H$-node distribution, based on a given distribution of $E$-nodes. The dashed polyhedrons are generated via a Voronoi tessellation.

when using local RBFs compared to global basis functions. This comes at a cost of reduced interpolation accuracy.

\section{NODE DistRIBUTION}

In contrast to second-order problems (e.g., wave equation) where only one field, electric or magnetic, needs to be calculated, special arrangements of the collocation nodes are advantageous when the Maxwell's equations in first-order form are solved. In the FDTD method, the staggered Yee scheme yields second-order accuracy [25]. A similar approach is taken in the present case, which results in two sets of staggered node distributions for $E$ - and $H$-fields. All components of the $E$-field are stored in one set of nodes ( $E$-nodes), and all components of the $H$-field are stored in a dual node distribution ( $H$-nodes) [26].

\section{A. Dual Node Distribution}

The generation of the two sets of staggered node distributions is done as follows: first an arbitrarily distributed set of $E$-nodes is generated. This facilitates modeling of the boundaries due to the currently implemented Dirichlet boundary conditions for the transverse electric field [perfect electric conductors (PECs)]. A Voronoi tessellation of these nodes [27] is generated and the $H$-nodes are placed on the edge centers of the Voronoi cells, as illustrated in Fig. 4. This leads to two separate sets of shape functions that approximate the $E$ - and $H$-field component values, respectively. The approximation for the scalar spatial derivatives $\partial \kappa$ of the $E$-field can be expressed as $\partial_{\kappa} \Psi_{E}\left(\mathbf{x}_{H}\right)$ at the $H$-node locations $\mathbf{x}_{H}$ in accordance with (7). Vice versa, for the dual nodes, $\partial_{\kappa} \boldsymbol{\Psi}_{H}\left(\mathbf{x}_{E}\right)$ are the shape functions for the spatial derivatives of the $H$-field at the $E$-node locations $\mathbf{x}_{E}$.

\section{B. Superposition}

If two nodes are anomalously close to each other in an otherwise nearly homogeneous node distribution, two almost identical entries in the interpolation matrix $\mathbf{G}$ (4) are created. This leads to ill conditioning, regardless of the shape parameter $\alpha_{c}$. Hence, in such cases, a matrix solver will always give inaccurate interpolation results. During node generation, a limit for the minimum relative node distance solves this issue.

\section{Boundaries}

It has been pointed out in [28] that interpolation accuracy is increased when a higher density of nodes is chosen close to 
and at the boundary. Thus, in the present case, node density for boundary nodes has been doubled.

\section{ApPlicATion to MaXwell's EQuations}

The discretized conservative and source-free Maxwell's equations can be written as

$$
\begin{aligned}
\text { frequency domain: } & j \omega \mathcal{M}\left(\begin{array}{l}
\mathbf{E} \\
\mathbf{H}
\end{array}\right)=\mathcal{L}\left(\begin{array}{l}
\mathbf{E} \\
\mathbf{H}
\end{array}\right) \\
\text { time domain: } & \partial_{t} \mathcal{M}\left(\begin{array}{l}
\mathbf{E} \\
\mathbf{H}
\end{array}\right)=\mathcal{L}\left(\begin{array}{l}
\mathbf{E} \\
\mathbf{H}
\end{array}\right)
\end{aligned}
$$

where $\mathcal{M}=\operatorname{diag}(\varepsilon, \mu)$ is the diagonal material mass matrix and $\mathcal{L}=\left(\begin{array}{cc}\mathbf{0} & \nabla \times \\ -\nabla \times & \mathbf{0}\end{array}\right)$ is the curl matrix. $\mathbf{E}$ and $\mathbf{H}$ denote the electric and magnetic vector fields.

\section{A. Eigenvalue Solver}

The eigenvalues correspond to the resonant frequencies $\omega=$ $-j \lambda$ of the resonator and the eigenvectors represent the field distribution of each mode. In a 2-D transverse magnetic (TM) case with the $z$-invariant field components $H_{x}, H_{y}$, and $E_{z}$, the problem is reduced to the generalized eigenvalue problem

$$
\lambda \mathcal{M}\left(\begin{array}{c}
\mathbf{E}_{z} \\
\mathbf{H}_{x} \\
\mathbf{H}_{y}
\end{array}\right)=\mathcal{L}^{h}\left(\begin{array}{c}
\mathbf{E}_{z} \\
\mathbf{H}_{x} \\
\mathbf{H}_{y}
\end{array}\right) .
$$

The vectors $\mathbf{E}_{z}, \mathbf{H}_{x}$, and $\mathbf{H}_{y}$ are vectors with lengths equal to the number of electric and magnetic field nodes, respectively. The matrix $\mathcal{L}^{h}$ for TM-polarized waves contains the spatial derivatives in the $x$ - and $y$ - direction

$$
\mathcal{L}^{h}=\left(\begin{array}{ccc}
\mathbf{0} & -\partial_{y} \mathbf{L}_{e} & \partial_{x} \mathbf{L}_{e} \\
-\partial_{y} \mathbf{L}_{h} & \mathbf{0} & \mathbf{0} \\
\partial_{x} \mathbf{L}_{h} & \mathbf{0} & \mathbf{0}
\end{array}\right) .
$$

The real valued matrices $\partial_{x} \mathbf{L}_{e}, \partial_{y} \mathbf{L}_{e}, \partial_{x} \mathbf{L}_{h}, \partial_{y} \mathbf{L}_{h}$ are obtained by inserting the derivatives of the shape functions (7)

$$
\partial_{\kappa} L_{e}(i, j)=\partial_{\kappa} \Psi e^{i}\left(\mathbf{x}_{H}(j)\right)
$$

and

$$
\partial_{\kappa} L_{h}(i, j)=\partial_{\kappa} \Psi h^{i}\left(\mathbf{x}_{E}(j)\right) .
$$

For local RBFs, these matrices are of sparse nature with the number of entries per row equal to the number of neighbors considered in the support domain.

The present investigation concerns cavity resonators with a perfect electric conducting boundary. Due to the delta property of RBF interpolation, this Dirichlet boundary is implemented by placing zero-valued $E$-nodes at the boundary.

\section{B. Time-Domain Solver}

A thorough description of the time-domain implementation used here has been given in [18]. Therefore, the method is only

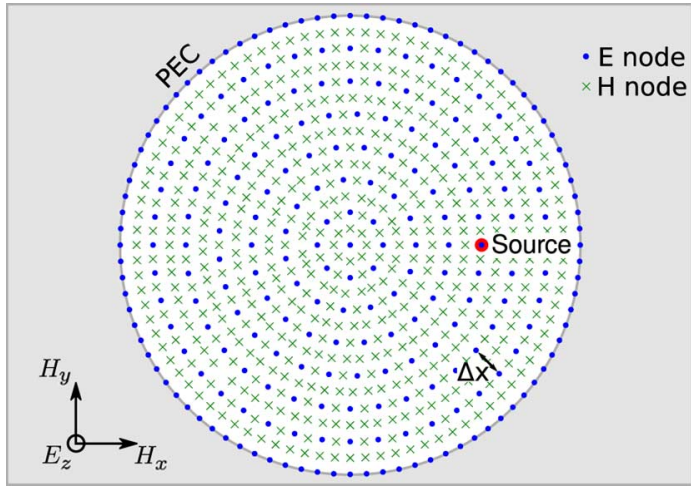

Fig. 5. Model of the cylindrical cavity with staggered $E$ - and $H$-node distributions.

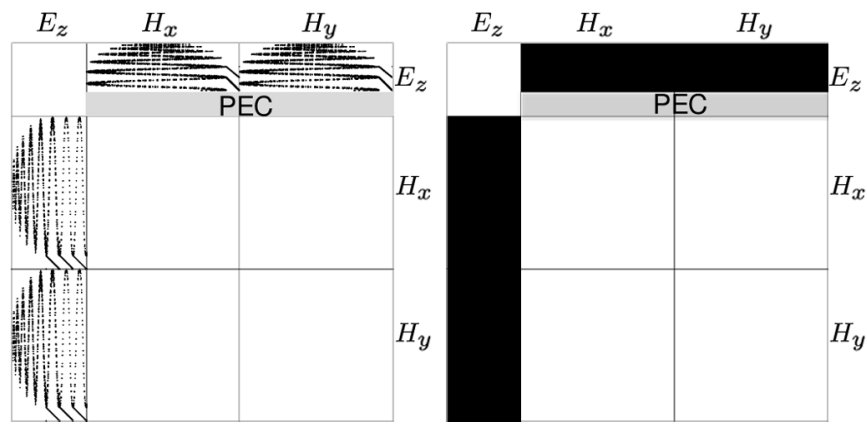

Fig. 6. Representation of the stiffness matrix $\mathcal{L}^{h}$ using: (left) local and (right) global basis functions. Nonzero elements are indicated by dots.

briefly summarized below. The temporal derivative in (9) is discretized by a staggered march in time, which corresponds to a second-order leapfrog scheme. This has been chosen here for simplicity and efficiency. When retaining the nomenclature of the previous section, the explicit time-domain update equations can be expressed as

$$
\begin{aligned}
\mathbf{E}_{z}^{(n+1 / 2)}= & \mathbf{E}_{z}^{(n-1 / 2)}+\frac{\Delta t}{\varepsilon_{0}}\left[\partial_{x} \mathbf{L}_{e} \mathbf{H}_{y}^{(n)}\right. \\
& \left.\quad-\partial_{y} \mathbf{L}_{e} \mathbf{H}_{x}^{(n)}-\mathbf{J}_{z}^{(n)}\right] \\
\mathbf{H}_{x}^{(n+1)}= & \mathbf{H}_{x}^{(n)}-\frac{\Delta t}{\mu_{0}} \partial_{y} \mathbf{L}_{h} \mathbf{E}_{z}^{(n+1 / 2)} \\
\mathbf{H}_{y}^{(n+1)}= & \mathbf{H}_{y}^{(n)}+\frac{\Delta t}{\mu_{0}} \partial_{x} \mathbf{L}_{h} \mathbf{E}_{z}^{(n+1 / 2)}
\end{aligned}
$$

with the source term $\mathbf{J}_{z}$ and superscripts $n$ indicating the index of the time step.

The stability region of the leapfrog scheme for first-order problems lies on the imaginary axis. The maximum imaginary part of the eigenvalues therefore determines the maximum time step for stable iterations. In [29], the authors apply the condition

$$
\Delta t \leq \min _{i} \frac{d_{\min _{i}}}{c_{0}}
$$

for the time stepping. This estimate is based on the distance $d_{\min _{i}}$ to the closest neighbor of node $i$ and the speed of light 


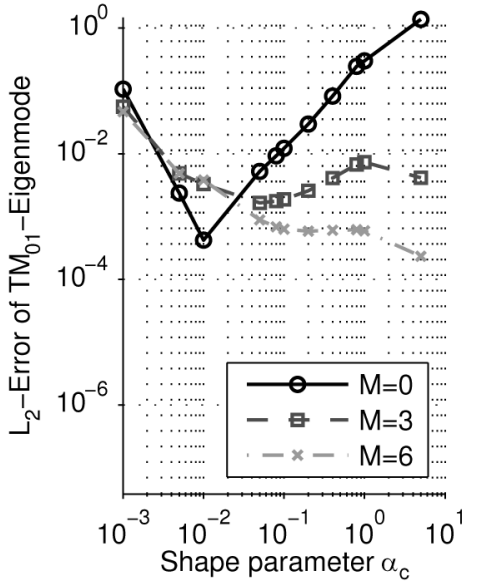

(a)

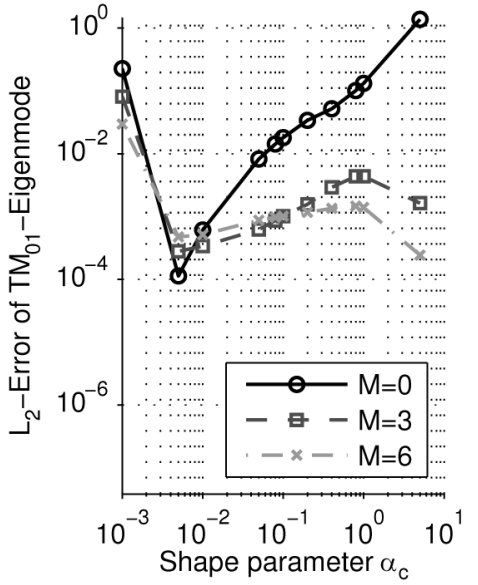

(b)

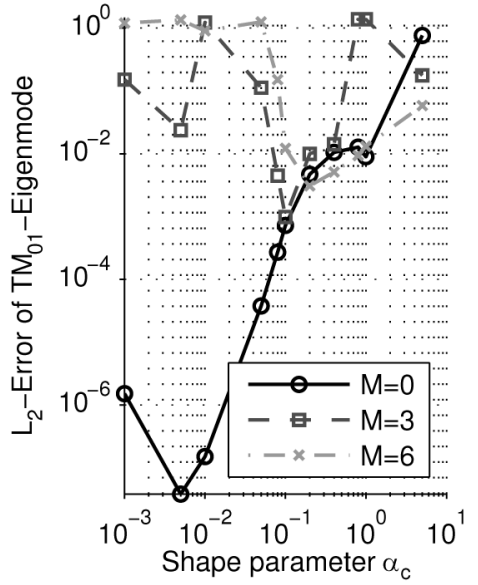

(c)

Fig. 7. Convergence analysis for the shape parameter $\alpha_{c}$. The $L_{2}$-error $\epsilon_{L_{2}}$ is shown for local RBF with $d_{s}=1.5 d_{c}$ in (a), $d_{s}=1.8 d_{c}$ in (b), and global RBFs in (c).

$c_{0}$. It proves to be a good, even though conservative estimate for stable simulations.

In the following numerical experiments, it is found that for some sets of parameters, the high-frequency eigenvalues have nonzero real parts. These eigenvalues correspond to nonphysical instabilities that become apparent after long simulation time (hundreds of periods). Several approaches can be taken to improve longtime stability.

1) Conditions for the interpolation parameters can be determined for which the eigenvalue distribution fits into the stability region of the leapfrog scheme in the form of a parameter study.

2) Reference [30] describes several high-order schemes that include nonzero real parts of the eigenvalue distribution into the stability region. The high-order schemes result, however, in higher computational costs and requires scaling of the time step to fit all nonzero valued eigenvalues into the stability region.

3) In [31], the implementation of a digital low-pass filter into the time iteration is proposed to suppress unstable modes for a hybrid finite-element/FDTD method. This approach unfortunately adds dispersion to the numerical solution.

In this paper, the first of these approaches is chosen. An extensive parametric study is conducted to find which parameters are suitable to provide longtime stability.

\section{NUMERICAL EXPERIMENTS}

Numerical experiments in a cylindrical PEC cavity have been performed. The discretized physical model of the cylindrical cavity is depicted in Fig. 5. The average node distance is $\Delta x$, and for the time-domain simulations, a source node is placed off-center. At the boundary, the node density is increased for higher accuracy. The cavity has a radius of $149 \mathrm{~mm}$, which corresponds to one free-space wavelength at $2 \mathrm{GHz}$. The stiffness matrix $\mathcal{L}^{h}(11)$ and the mass matrix $\mathcal{M}(9)$ have been assembled before solving the eigenvalue problem (10). The stiffness matrix configuration is depicted in Fig. 6 for local and global basis functions. The localized case results in a sparse matrix. For global basis functions, the connectivity of all nodes results in a block-wise full matrix. For the corresponding time-domain simulations, the explicit update equations (14) are used.

In a first step, numerical experiments are performed for the eigenvalue problem to show parameter convergence and spatial convergence. The analysis in [20], which was focused on the relative errors for the calculated resonance frequency, is extended here to quantify the error in the $\mathrm{TM}_{01}, \mathrm{TM}_{02}$ field distributions. The $L_{2}$-error between the numerically computed $E$-field $E^{\mathcal{N}}$ and the theoretical field $E^{\text {th }}$ is calculated as

$$
\begin{aligned}
\epsilon_{L_{2}} & =\sqrt{\frac{\int_{\Omega}\left|E_{z}^{\mathcal{N}}-E_{z}^{\mathrm{th}}\right|^{2} d x}{\int_{\Omega}\left|E_{z}^{\mathrm{th}}\right|^{2} d x}} \\
& \approx \sqrt{\frac{\sum_{i}\left|E_{z}^{\mathcal{N}}(i)-E_{z}^{\mathrm{th}}(i)\right|^{2} A(i)}{\sum_{i}\left|E_{z}^{\mathrm{th}}\right|^{2} A(i)}}
\end{aligned}
$$

where $\Omega$ denotes the computational domain. The domain $\Omega$ is fragmented into Voronoi cells during the generation of the dual grid that defines an area $A(i)$ associated to each $E$-node $i$. The relative eigenvalue error has been calculated as

$$
\epsilon_{\mathrm{rel}}=\frac{\left|\frac{\lambda}{2 \pi j}-f_{m n}^{\mathrm{th}}\right|}{f_{m n}^{\mathrm{th}}}
$$

with $\lambda$ being the complex valued numerical eigenvalue. The exact $(m, n)$-eigenvalues and $(m, n)$-eigenmodes are

$$
\begin{aligned}
f_{n m}^{\mathrm{th}} & =\left(\frac{p_{n m}}{r}\right) \frac{c_{0}}{2 \pi} \\
E_{z, m n}^{\mathrm{th}}(\rho, \phi) & =J_{m}\left(\rho \frac{p_{n m}}{r}\right) \cos (m \phi)
\end{aligned}
$$

where $p_{m n}$ denotes the $n$th zero of the $m$ th-order Bessel function $J_{m}\left(p_{m n}\right)=0$ and $c_{0}$ stands for the speed of light in free space [32].

In a second step, the total energy in the system has been observed for time-domain simulations. The explosion of nonphysical modes can be linked to nonzero real parts of the eigenvalues. As a lossless resonant cavity is simulated, the energy in the system has to remain constant after having been introduced 


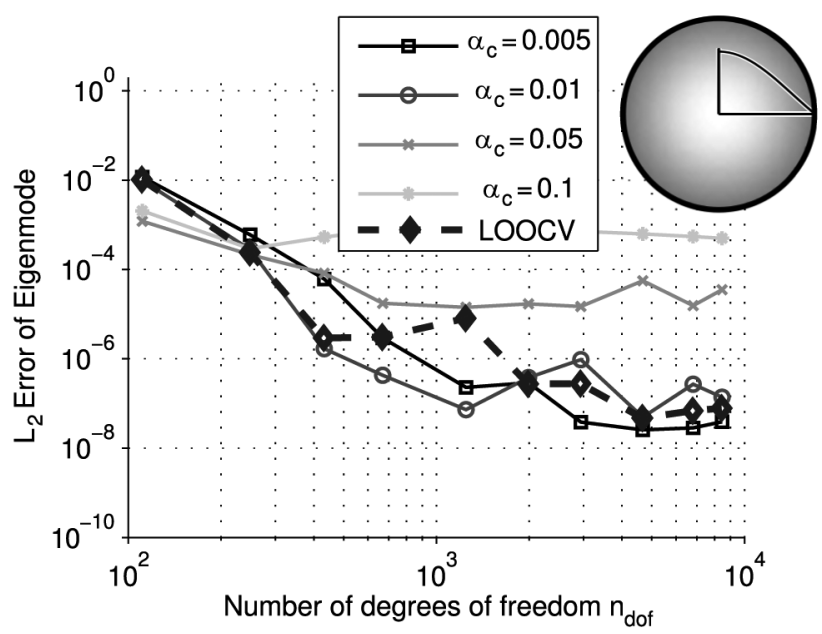

(a)

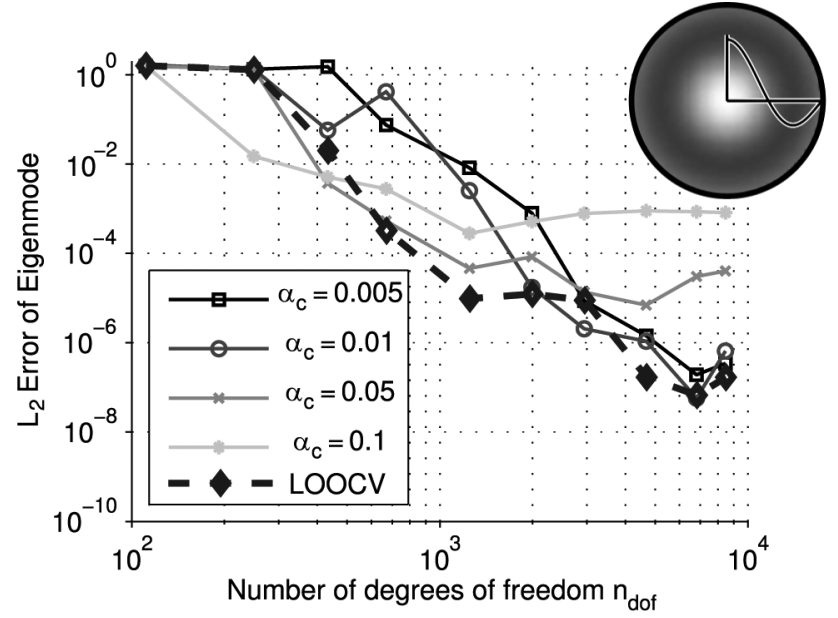

(b)

Fig. 8. Convergence study of two eigenmode field distributions $\mathrm{TM}_{01}$ in (a) and $\mathrm{TM}_{02}$ in (b) for the $E$-field for global RBFs. The $L_{2}$-error $\epsilon_{L_{2}}$ is calculated for different shape parameters $\alpha_{c}$.

into the system. Observing the total energy allows to investigate longtime stability and possible dissipation effects. The normalized energy of the discretized problem is approximated as

$$
\mathcal{E} \approx \varepsilon_{0} \sum_{i}\left|E_{z}^{\mathcal{N}}(i)\right|^{2}+\mu_{0} \frac{N_{H}}{N_{E}} \sum_{i}\left(\left|H_{x}^{\mathcal{N}}(i)\right|^{2}+\left|H_{y}^{\mathcal{N}}(i)\right|^{2}\right)
$$

assuming a homogeneous node distribution with approximately equal areas of each Voronoi cell. The ratio $N_{H} / N_{E}$ of number of $H$ - and $E$-nodes estimates the size difference between $E$-and $H$-cell areas. The approximation is justified for the present problem, as the $E_{z}$-field is zero at the boundary, where the node distribution is inhomogeneous.

\section{A. Parameter Convergence}

To analyze parameter convergence, a node distribution with fixed discretization of $\Delta x=12.5 \mathrm{~mm}$ or 12 nodes over the radius has been generated. This results in a total of $n_{\text {dof }}=2944$ degrees of freedom. The convergence of the first eigenmode $\left(\mathrm{TM}_{01}\right)$ was analyzed in terms of parameter selection. The results are expected to yield better accuracy for small shape parameters $\alpha_{c}$, i.e., flat RBFs, until at a certain point the matrix condition number becomes too high for accurate numerical inversion and the interpolation breaks down. A comparison is performed between local and global RBFs. For the local case, two different support domain sizes have been chosen, resulting in $N=10.4$ neighbors on average for $d_{s}=1.5 d_{c}$ and $N=16.5$ for $d_{s}=1.8 d_{c}$. The $L_{2}$-error has been calculated and is shown in Fig. 7. When no monomial basis functions are used $(M=0)$, it can be observed that a higher accuracy is achieved with global RBFs compared to local RBFs. The additional monomial basis functions $(M>0)$ prove beneficial for local basis functions as the shape parameter $\alpha_{c}$ is increased. The expected increased accuracy due to a reduced shape parameter $\alpha_{c}$ could generally be confirmed. However, this happens at the cost of stability, as expressed by the breakdown in accuracy observed for the smallest shape parameters.

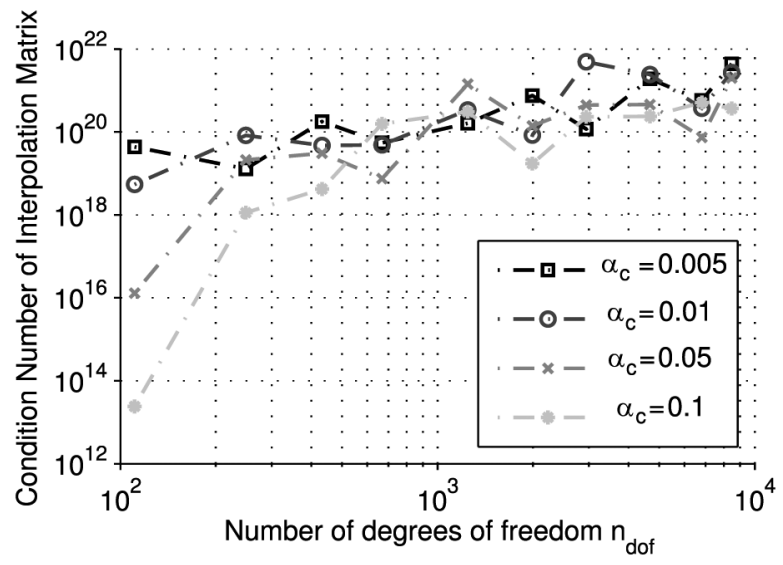

Fig. 9. Condition number for the parameter sets in Fig. 8.

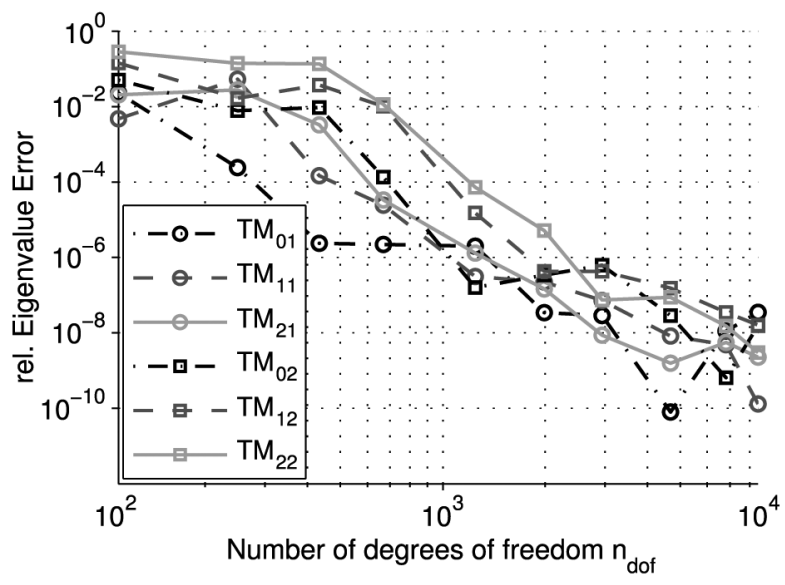

Fig. 10. Spatial convergence of the first six eigenvalues. The relative eigenvalue error $\epsilon_{\mathrm{rel}}$ is calculated for the eigenmodes $\mathrm{TM}_{01} \ldots \mathrm{TM}_{22}$.

For global RBFs with monomial reproduction $(M>0)$, it is not possible to reach results comparable to $M=0$ because the influence of an increased condition number impairs the results more severely than the gain in accuracy. It can be finally 
TABLE I

Comparison of Number of Degrees of FreEdom $\left(n_{\text {dof }}\right)$ AND EXeCUtion Time Between the FIRST-ORDER FEM, SECOND-ORDER FDs, AND RPIM TO ACHIEVE A GIVEN ACCURACY

\begin{tabular}{|c|c|r|r|r|r|r|r|}
\hline $\begin{array}{c}\text { Eigenvalue } \\
\text { Error }\end{array}$ & \multirow{2}{*}{ Mode } & \multicolumn{2}{|c|}{ FD } & \multicolumn{2}{c|}{ FEM } & \multicolumn{2}{c|}{ RPIM } \\
& & \multicolumn{1}{|c|}{$n_{\text {dof }}$} & time & \multicolumn{1}{c|}{$n_{d o f}$} & \multicolumn{1}{c|}{ time } & $n_{d o f}$ & time \\
\hline \multirow{2}{*}{$<10^{-3}$} & $T M_{01}$ & $1.52 \cdot 10^{6}$ & $71.6 \mathrm{~s}$ & 1079 & $4.34 \mathrm{~s}$ & 205 & $0.12 \mathrm{~s}$ \\
& $T M_{22}$ & $1.61 \cdot 10^{6}$ & $76.4 \mathrm{~s}$ & $4.12 \cdot 10^{4}$ & $12.35 \mathrm{~s}$ & 947 & $0.91 \mathrm{~s}$ \\
\hline \multirow{2}{*}{$<10^{-6}$} & $T M_{01}$ & - & - & $1.03 \cdot 10^{6}$ & $436.94 \mathrm{~s}$ & 1393 & $2.03 \mathrm{~s}$ \\
& $T M_{22}$ & - & - & - & - & 2358 & $6.84 \mathrm{~s}$ \\
\hline
\end{tabular}

observed that when the size of the local support domain is increased, the results approach those achieved using global support.

For practical implementations, depending on the required accuracy, either global RBFs without monomial basis functions $M=0$ and with a coarse discretization, or local basis functions with monomial expansion $M>0$ using finer discretizations, can lead to efficient simulations.

\section{B. Spatial Convergence Using Global Basis Functions}

In [20], the spatial eigenvalue convergence for local basis functions has been demonstrated. Here, these findings are supplemented by analyzing the performance of global basis functions. Monomial reproduction has been shown not to be beneficial for global basis functions in the convergence analysis of the previous section, therefore it is not applied in the following $(M=0)$. Several node distributions with discretizations in the range of $[50 \mathrm{~mm}, \ldots, 7.5 \mathrm{~mm}]$ are considered, which correspond to discretizations of $[3, \ldots, 20]$ nodes per radius. This results in $[111, \ldots, 8457]$ numbers of degrees of freedom. We use a sparse eigensolver as provided by ARPACK [33] to compute a few eigenvalues of these large systems.

1) Parameter Influence: In a first step, the $L_{2}$-error of the $\mathrm{TM}_{01}$ and $\mathrm{TM}_{02}$ eigenmodes have been calculated and the results are depicted in Fig. 8. The $L_{2}$-error converges at different rates for different shape parameters. Generally, lower values for the shape parameter $\alpha_{c}$, i.e., increasingly flat basis functions, give higher accuracy. High convergence can then be achieved by an optimization of the shape parameter, for a given node distribution [12]. Therefore, the "leave-one-out cross validation" (LOOVC) algorithm [8] has been implemented, which optimizes the shape parameter $\alpha_{c}$ for maximum accuracy by using a minimization algorithm. The cost function is an efficient approximation of the maximum error that is built by removing one node at a time and calculating the interpolation error for the derivative at this node. The LOOCV algorithm gives a good approximation of the optimized shape parameter.

Fig. 9 shows the two-norm condition number of the interpolation matrix. Best results were achieved when the condition number is as high as possible while remaining below the limit where the accuracy of the matrix inversion breaks down. The condition number yields degenerated estimates for very ill-conditioned matrices with cond $(\mathbf{G})>10^{21}$. Thus, only unreliable conclusions can be drawn above this value even if highly accurate interpolation accuracy might still be achieved for very high condition numbers.

2) Optimizing Shape Parameter: In a second step, the convergence of the relative eigenvalue error has been investigated for the eigenmodes $\mathrm{TM}_{01} \ldots \mathrm{TM}_{22}$. Each discretization is
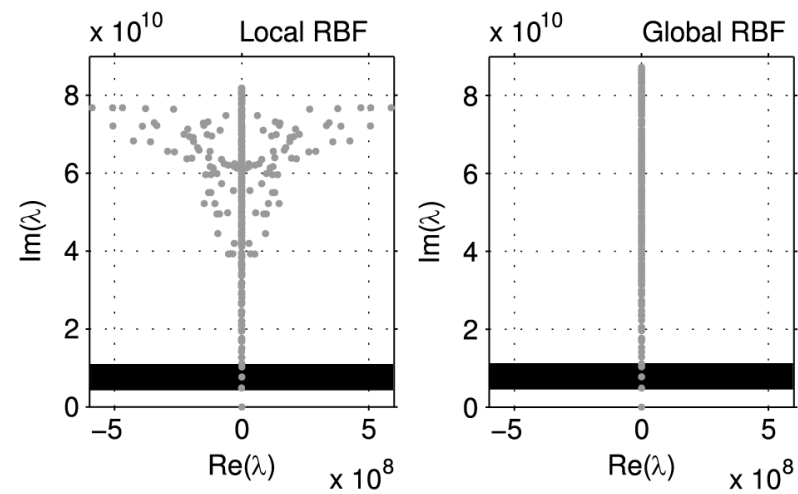

Fig. 11. Eigenvalue distribution for the parameter set $\alpha_{c}=0.4, M=3$. On the left are local RBFs with a support domain size of $d_{s}=1.8 d_{c}$, on the right are global RBFs. The dark bar represents the bandwidth of the time-domain simulation.

again subject to an optimization of the shape parameter by the LOOCV algorithm. The results are shown in Fig. 10, demonstrating the convergence for all presented eigenvalues. Table I compares the execution time for the eigenvalue solvers of RPIM versus the finite-element and finite-differences toolbox in MATLAB. The times are given for two cases that consider different accuracies in terms of the $L_{2}$-errors. The comparison demonstrates the advantages of the highly accurate RBF interpolation technique. Note that only conventional $h$-FEM is examined. High convergence rates can also be achieved by using $h p$-FEM with curved elements [11]. The difficulties for finite differences (FDs) to achieve the required accuracy is explained by the low-order in-cell approximation and stair-casing effects.

\section{Eigenvalue Distribution}

The eigenvalue distribution computed using an eigenvalue solver for full matrices [34] is plotted in Fig. 11. The eigenvalues are symmetric with respect of the imaginary and real axis. It can be observed first that dc modes, i.e., at $\omega=0$, exist. This is not surprising as the divergence condition of Maxwell's equations is not explicitly enforced. Further, at low frequencies, no unphysical modes are observed, i.e., the eigenvalues are purely imaginary and each corresponds to a physical mode. At higher frequencies, nonzero real parts begin to occur. Even if these unstable eigenmodes are at very high frequencies, beyond the highest simulated frequency in time-domain solutions, eigenvalues with nonzero real parts are known to cause instabilities in time-domain simulations: due to numerical noise, the modes get excited and eventually cause instabilities because of their nonphysical amplification. 


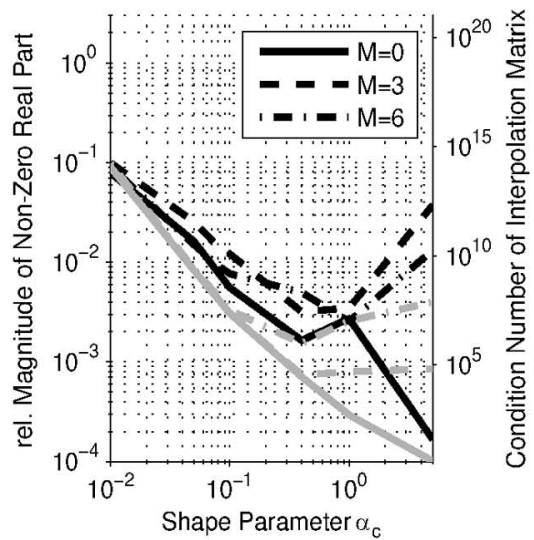

(a)

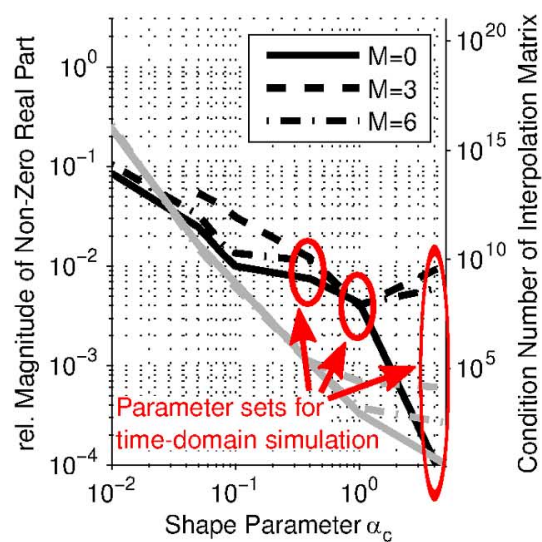

(b)

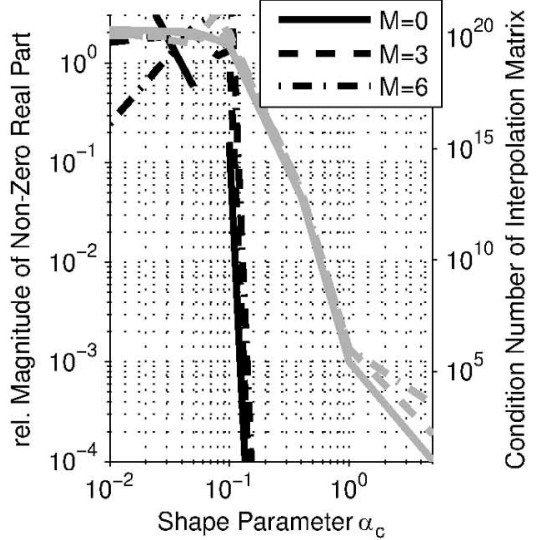

(c)

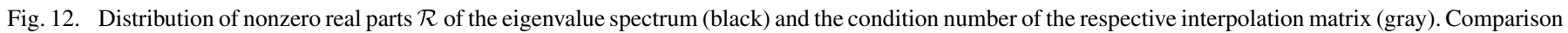
between local RBF with: (a) $d_{s}=1.5 d_{c}$ (b), local RBF with $d_{s}=1.8 d_{c}$, and (c) global RBFs.

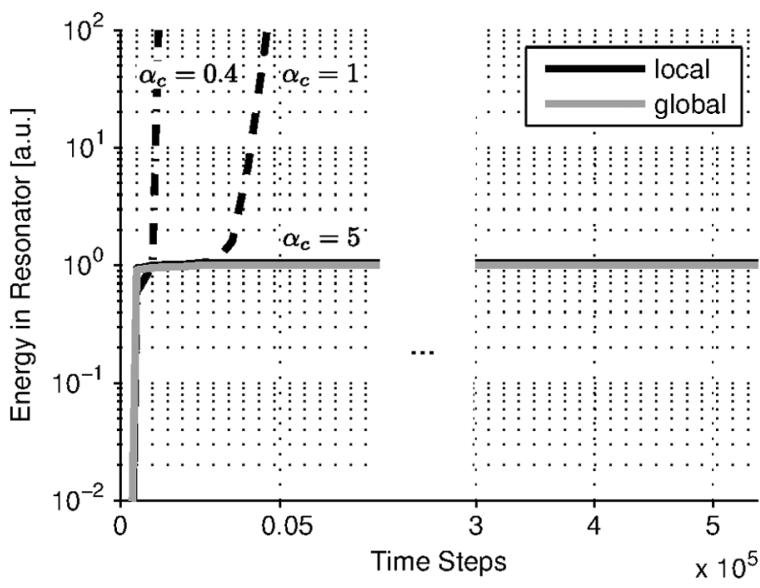

(a)

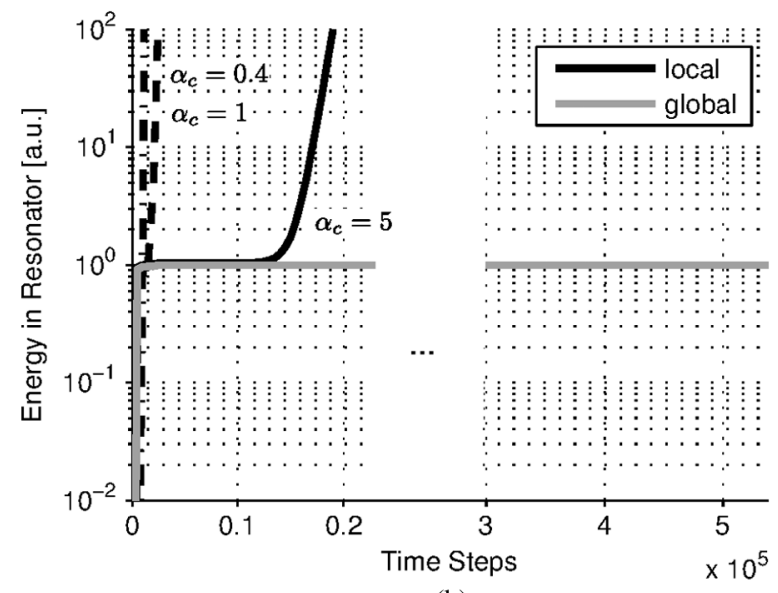

(b)

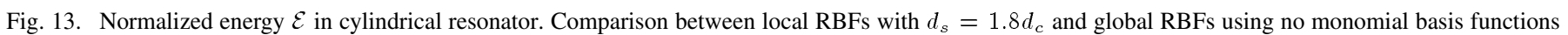
$(M=0)$ in (a) and first-order monomial basis functions $(M=3)$ in (b).

The magnitudes of these nonzero real parts have been analyzed for multiple sets of parameters and the relative magnitude is calculated according to

$$
\mathcal{R}=\max _{i} \frac{\operatorname{Re}\left\{\lambda_{i}\right\}}{\operatorname{Im}\left\{\lambda_{i}\right\}} .
$$

Fig. 12 shows these magnitudes for local and global RBFs. With local basis functions and no monomial basis functions $(M=0)$, the magnitude of the nonzero real parts decreases for increasing shape parameter $\alpha_{c}$, which is indicative of a longer time stability. For $M>0$ though, the magnitudes $\mathcal{R}$ remain quite high, with a minimum at approximately $\alpha_{c}=1$. For global RBFs, the magnitude of the nonzero real part goes to zero, when the matrix condition number is significantly below the numerical limit of $10^{21}$ at $\alpha_{c} \geq 0.1$. This means that time-domain simulations will always be longtime stable, provided the condition number is sufficiently low. This also applies to all cases with a monomial basis function. When the size of the support domain is increased for local RBFs, the condition number of the interpolation matrix is driven up, but the nonzero real parts decrease in the region where $\operatorname{cond}(G)<10^{21}$.

\section{Time Domain}

In the following, time-domain simulations are performed for very long times to relate the previous findings on spurious modes to long-term stability. The same node distribution as for the parameter convergence has been chosen with 2944 degrees of freedom. Energy was injected into the system over one source node in the bandwidth $f=[1 \mathrm{GHz}, 2 \mathrm{GHz}]$. The simulation has been run for 10000 periods at the center frequency. The number of time steps required was $N=535,490$. In Fig. 13, the energy over time is shown for different parameters. Local RBFs with $d_{s}=1.8 d_{c}$ are compared against global RBFs. It is clearly observed that the higher the nonzero real part of the eigenvalue, the earlier instabilities occur. In the case of local RBFs, for higher values of the shape parameter $\alpha_{c} \geq 5$, this magnitude becomes very small and longtime stability is ensured. As expected, global RBFs perform very well for $\alpha_{c} \geq 0.1$. For smaller values of $\alpha_{c}$, nonphysical modes dominate the time-domain simulations from early on. It is also noted that no dissipative effects have been observed for any set of parameters. 


\section{CONCLUSION}

A detailed study of the eigenvalue properties of the RPIM for electromagnetics with a staggered $E$ - and $H$-node distribution has been performed. For a fixed discretization, the numerical error can be reduced by optimizing the shape parameter ( $c$-refinement). The error decreases for increasingly flat basis functions, until the numerical limit of the interpolation matrix inversion is reached. Due to theoretical exponential convergence, global RBFs allow for much smaller errors than local basis functions at the cost of increased computational effort associated with the inversion of a full system matrix in preprocessing. Due to the spatial convergence, accuracy can also be increased by refined spatial discretization ( $h$-refinement). Again operating at high matrix condition numbers, the errors decrease for increasingly fine discretizations. By applying the LOOCV algorithm, a shape parameter could automatically be selected. This suggests the need for further research in the field of $h c$-adaptivity as the combination of the two refinements appears the most efficient strategy.

The relative magnitudes of the nonzero real parts of the eigenvalues have been confirmed as an indicator for the longtime-stability of time-domain simulations. Local basis functions with low monomial degree and large shape parameters, or global basis functions with sufficiently large shape parameters, proved to be a feasible solution to grant stable simulations over very long times. In general, global RBFs can be applied for small problems with high accuracy, or in combination with a domain decomposition method leading to block-wise full matrices for large problems. If the problem becomes very complex, local RBFs can be used to resolve the geometry with a large number of points.

In future work, for a time-domain implementation, the accuracy of the time integration can be improved by using high-order time-stepping schemes. In general, the method can be further improved with a better optimization algorithm for the shape parameter. For practical implementations, the treatment of material discontinuities has to be further addressed to reduce the Gibbs phenomena due to discontinuities in the fields at material interfaces.

\section{REFERENCES}

[1] S. A. Viana, D. Rodger, and H. C. Lai, "Overview of meshless methods," ICS Newslett., vol. 14, no. 2, pp. 3-6, Nov. 2007.

[2] T. Belytschko, Y. Krongauz, D. Organ, M. Fleming, and P. Krysl, "Meshless methods: An overview and recent developments," Comput. Methods Appl. Mech. Eng., vol. 139, no. 1-4, pp. 3-47, 1996.

[3] A. Angulo, L. P. Pozo, and F. Perazzo, "A posteriori error estimator and an adaptive technique in meshless finite points method," Eng. Anal. Boundary Elements, vol. 33, no. 11, pp. 1322-1338, 2009.

[4] J. J. Monaghan, "Smoothed particle hydrodynamics," Annu. Rev. Astron. Astrophys., vol. 30, pp. 543-574, 1992.

[5] X. Zhang, X.-H. Liu, K.-Z. Song, and M.-W. Lu, "Least-squares collocation meshless method," Int. J. Numer. Methods Eng., vol. 51, no. 9, pp. 1089-1100, 2001.

[6] E. Kansa, "Multiquadric scattered data approximation scheme with applications to computational fluid dynamics-II: Solutions to parabolic, hyperbolic and elliptic partial differential equations," Comput. Math. Appl., vol. 19, no. 8-9, pp. 147-161, 1990.

[7] G. Liu and Y. Gu, An Introduction to Meshfree Methods and Their Programming. Dortrecht, The Netherlands: Springer, 2005.

[8] G. F. Fasshauer, Meshfree Approximation Methods With MATLAB. River Edge, NJ: World Sci., 2007.
[9] G. R. Liu and Y. T. Gu, "A local radial point interpolation method (LRPIM) for free vibration analyses of 2-D solids," J. Sound Vib., vol. 246, no. 1, pp. 29-46, Sep. 2001.

[10] W. Madych, "Miscellaneous error bounds for multiquadric and related interpolators," Comput. Math. Appl., vol. 24, no. 12, pp. 121-138, 1992.

[11] I. Babuška and M. Suri, "The $p$ and $h-p$ versions of the finite element method, basic principles and properties," Soc. Indus. Appl. Math. Rev., vol. 36, no. 4, pp. 578-632, 1994.

[12] A. H.-D. Cheng, M. A. Golberg, E. J. Kansa, and G. Zammito, "Exponential convergence and $h-c$ multiquadric collocation method for partial differential equations," Numer. Methods Partial Diff. Equations, vol. 19 , no. 5, pp. 571-594, 2003.

[13] G. Ala, E. Francomano, A. Tortorici, E. Toscano, and F. Viola, "Smoothed particle electromagnetics: A mesh-free solver for transients," J. Comput. Appl. Math., vol. 191, no. 2, pp. 194-205, 2006.

[14] K. Krohne, G.-H. Park, and L. E. Ping, "A two-dimensional smoothed particle time-domain method," in Asia-Pacific Microw. Conf., Dec. 2008, pp. 1-4.

[15] T. Kaufmann, C. Fumeaux, and R. Vahldieck, "The meshless radial point interpolation method for time-domain electromagnetics," in IEEE MTT-S Int. Microw. Symp. Dig., Atlanta, GA, Jun. 2008, pp. 61-65.

[16] Y. Yu and Z. D. Chen, "An unconditionally stable radial point interpolation method for efficient meshless modeling in time domain," in 25th Int. Rev. Progr. Appl. Comput. Electromagn., Monterey, CA, Mar. 2009, pp. 389-394.

[17] Y. Yu and Z. D. Chen, "A 3-D radial point interpolation method for meshless time-domain modeling," IEEE Trans. Microw. Theory Tech., vol. 57, no. 8, pp. 2015-2020, Aug. 2009.

[18] T. Kaufmann, C. Fumeaux, and R. Vahldieck, "Parameter selection for the radial point interpolation time-domain method," in 25th Int. Rev. Progr. Appl. Comput. Electromagn., Monterey, CA, Mar. 2009, pp. 395-400.

[19] S. Lai, B. Wang, and Y. Duan, "Meshless radial basis function method for transient electromagnetic computations," IEEE Trans. Magn., vol. 44, no. 10, pp. 2288-2295, Oct. 2008.

[20] T. Kaufmann, C. Fumeaux, C. Engstrom, and R. Vahldieck, "Meshless eigenvalue analysis for resonant structures based on the radial point interpolation method," in Asia-Pacific Microw. Conf., Dec. 2009, pp. 818-821.

[21] M. D. Buhmann, Radial Basis Functions. New York: Cambridge Univ. Press, 2003.

[22] G. Liu, Mesh Free Methods: Moving Beyond the Finite Element Method. Boca Raton, FL: CRC, 2009.

[23] G. Fasshauer and J. Zhang, "On choosing "optimal" shape parameters for RBF approximation," Numer. Algorithms, vol. 45, no. 1, pp. 345-368, Aug. 2007.

[24] G. E. Fasshauer and J. G. Zhang, "Preconditioning of radial basis function interpolation systems via accelerated iterated approximate moving least squares approximation," Comput. Methods Appl. Sci., vol. 11, pp. 57-75, 2009.

[25] A. Taflove and S. C. Hagness, Computational Electrodynamics: The Finite-Difference Time-Domain Method,3rd ed. Norwood, MA: Artech House, 2005.

[26] J.-F. Lee, R. Lee, and A. Cangellaris, "Time-domain finite-element methods," IEEE Trans. Antennas Propag., vol. 45, no. 3, pp. 430-442, Mar. 1997.

[27] C. B. Barber, D. P. Dobkin, and H. Huhdanpaa, "The quickhull algorithm for convex hulls," ACM Trans. Math. Softw., vol. 22, no. 4, pp. 469-483, 1996.

[28] B. Fornberg, T. A. Driscoll, G. Wright, and R. Charles, "Observations on the behavior of radial basis function approximations near boundaries," Comput. Math. Appl., vol. 43, no. 3, pp. 473-490, Feb. 2002.

[29] T. Kaufmann, C. Fumeaux, and R. Vahldieck, "Application of the radial point interpolation method as meshless time-domain technique in electromagnetics," in 24th Int. Rev. Progr. Appl. Comput. Electromagn., Niagara Falls, ON, Canada, Apr. 2008, pp. 426-431.

[30] M. Ghrist, B. Fornberg, and T. A. Driscoll, "Staggered time integrator for wave equations," Soc. Ind. Appl. Math. J. Numer. Anal., vol. 38, no. 3 , pp. 718-741, 2000.

[31] C.-T. Hwang and R.-B. Wu, "Treating late-time instability of hybrid finite-element/finite-difference time-domain method," IEEE Trans. Antennas Propag., vol. 47, no. 2, pp. 227-232, Feb. 1999.

[32] C. A. Balanis, Advanced Engineering Electromagnetics. New York: Wiley, 1989. 
[33] R. Lehoucq, D. Sorensen, and C. Yang, ARPACK Users' Guide: Solution of Large-Scale Eigenvalue Problems With Implicitly Restarted Arnoldi Methods. Philadelphia, PA: SIAM, 1998.

[34] E. Anderson, Z. Bai, C. Bischof, S. Blackford, J. Demmel, J. Dongarra, J. D. Croz, A. Greenbaum, S. Hammarling, A. McKenney, and D. Sorensen, LAPACK Users' Guide, 3rd ed. Philadelphia, PA: SIAM, 1999.

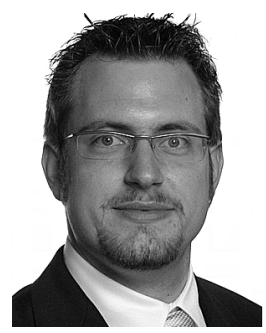

Thomas Kaufmann (S'06) received the M.Sc. degree in electrical engineering and information technology from ETH Zürich, Zürich, Switzerland, in 2007 , and is currently working toward the Ph.D. degree at the Laboratory for Electromagnetic Fields and Microwave Electronics (IFH), ETH Zürich.

During his doctoral studies, he visited the Laboratory of Sciences and Materials for Electronics and Automation (LASMEA), University Blaise Pascal, Clermont-Ferrand, France, the Institute for High Performance Computing, A*STAR, Singapore, and the School of Electrical and Electronic Engineering, The University of Adelaide, Australia, some among them for extended stays. His research interest includes computational electromagnetics for microwave circuits and antennas, particularly the novel field of meshless methods.

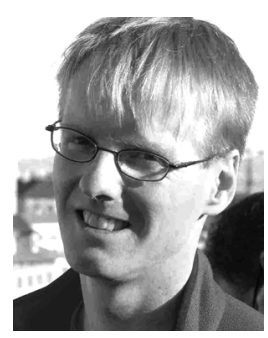

Christian Engström received the Masters degree in mathematics from Växjö University, Växjö, Sweden, in 1998, and the Ph.D. degree in electrical engineering from Lund University, Lund, Sweden, in 2006.

From 2007 to 2008, he was a Post-Doctoral Researcher with the Department of Mathematics, Karlsruhe Institute of Technology, Karlsruhe, Germany. Since 2009, he has been a Research Associate with the Laboratory for Electromagnetic Fields and Microwave Electronics (IFH), ETH Zürich, Zürich, Switzerland. His main research interests include computational electromagnetics and effective material models.

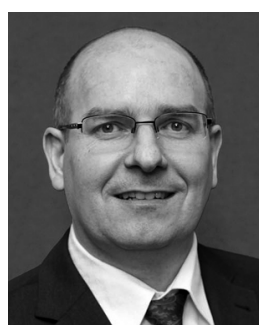

Christophe Fumeaux (M'03-SM'09) received the Diploma and Ph.D. degrees in physics from the ETH Zürich, Zürich, Switzerland, in 1992 and 1997, respectively.

From 1998 to 2000, he was a Postdoctoral Researcher with the School of Optics, University of Central Florida, Orlando. In 2000, he joined the Swiss Federal Office of Metrology, Bern, Switzerland, as a Scientific Staff Member. From 2001 to 2008, he was a Research Associate and Group Leader with the Laboratory for Electromagnetic Fields and Microwave Electronics (IFH), ETH Zürich. During Fall 2005, he was a Visiting Scientist with the Laboratory of Sciences and Materials for Electronics and
Automation (LASMEA), University Blaise Pascal, Clermont-Ferrand, France. In 2008, he joined the School of Electrical and Electronic Engineering, The University of Adelaide, Adelaide, Australia, as an Associate Professor. His current main research interest concerns computational electromagnetics, antenna engineering, terahertz technology, and the modeling of optical microstructures/nanostructures.

Dr. Fumeaux was the chairman of the IEEE Swiss Joint Chapter on Microwave Theory and Techniques (MTT-S), Antennas and Propagation (AP-S), and Electromagnetic Compatibility (EMC) from 2006 to 2008. Since 2010, he has been the chairman of the IEEE South Australia AP-S/MTT-S Chapter. He was the recipient of the ETH Silver Medal of Excellence for his doctoral dissertation. He was the corecipient of the 2004 Outstanding Paper Award of the Applied Computational Electromagnetics Society (ACES).

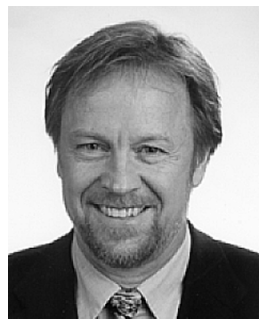

Rüdiger Vahldieck (M'85-SM'86-F'99) received the Dipl.-Ing. and Dr.-Ing. degrees in electrical engineering from the University of Bremen, Bremen, Germany, in 1980 and 1983, respectively.

Until 1986, he was a Postdoctoral Fellow with the University of Ottawa, Ottawa, ON, Canada. In 1986, he joined the Department of Electrical and Computer Engineering, University of Victoria, Victoria, BC, Canada, where he became a Full Professor in 1991. During Fall and Spring 1992 to 1993, he was a Visiting Scientist with the Ferdinand-Braun-Insitute für Hochfrequenztechnik, Berlin, Germany. In 1997, he became a Professor of electromagnetic field theory with the Swiss Federal Institute of Technology, Zürich, Switzerland, and in 2003, became Head of the Laboratory for Electromagnetic Fields and Microwave Electronics (IFH). In 2005, he became President of the Research Foundation for Mobile Communications and was elected Head of the Department of Information Technology and Electrical Engineering (D-ITET), ETH Zürich. Since 1981, he has authored or coauthored over 300 technical papers in books, journals, and conferences. His research interests include computational electromagnetics in the general area of electromagnetic compatibility (EMC), particularly for computer-aided design of microwave, millimeter wave and opto-electronic integrated circuits.

Dr. Vahldieck is the past-president of the IEEE 2000 International Zürich Seminar on Broadband Communications (IZS2000). Since 2003, he has been president and general chairman of the International Zürich Symposium on Electromagnetic Compatibility (EMC Zürich). He is a member of the Editorial Board of the IEEE TRansactions on Microwave Theory and TeChniques. From 2000 to 2003, he was associate editor for the IEEE MICROWAVE AND WIRELESS COMPONENTS LETTERS, and from July 2003 to the end of 2005, he was the editor-in chief. Since 1992, he has been on the Technical Program Committee of the IEEE Microwave Theory and Techniques Society (IEEE MTT-S) International Microwave Symposium (IMS), the IEEE MTT-S Technical Committee on Microwave Field Theory, and in 1999, on the Technical Program Committee (TPC) of the European Microwave Conference. From 1998 to 2003, he was the chapter chairman of the IEEE Swiss Joint Chapter on Microwave Theory and Techniques (MTT-S), Antennas and Propagation (AP-S), and Electromagnetic Compatibility (EMC). He was the recipient of the Outstanding Publication Award of the Institution of Electronic and Radio Engineers in 1983, the K. J. Mitra Award of the Institution of Electronics and Telecommunication Engineers (IETE) in 1996 for the best research paper of 1995, and the Applied Computational Electromagnetics Society (ACES) Outstanding Paper Award in 2004. 